\title{
Gene deletions in spinal muscular atrophy
}

Nanda R Rodrigues, Nicholas Owen, Kevin Talbot, Shobhna Patel, Francesco Muntoni, Jaakko Ignatius, Victor Dubowitz, Kay E Davies

\begin{abstract}
Two candidate genes (NAIP and SMN) have recently been reported for childhood onset spinal muscular atrophy (SMA). Although affected subjects show deletions of these genes, these deletions can lead to either a very mild or a severe phenotype. We have analysed a large number of clinically well defined patients, carriers, and normal controls to assess the frequency and extent of deletions encompassing both of these genes. A genotype analysis indicates that more extensive deletions are seen in the severe form of SMA than in the milder forms. In addition, $1.9 \%$ of phenotypically normal carriers are deleted for the NAIP gene; no carriers were deleted for the SMN gene. Our data suggest that deletions in both of these genes, using the currently available assays, are associated with both a severe and very mild phenotype.

(f Med Genet 1996;33:93-96)
\end{abstract}

Genetics Laboratory, Department of Genetics,

University of Oxford, South Parks Road, Oxford

OX1 3QU, UK

N R Rodrigues

$\mathrm{N}$ Owen

K Talbot

$S$ Patel

K E Davies

Neuromuscular Unit and Department of Paediatrics, Royal Postgraduate Medical School, Hammersmith Hospital,

Du Cane Road, London W12 0NN, UK $S$ Patel

F Muntoni

V Dubowitz

Department of Medical Genetics, University of Helsinki, Haartmaninkatu 3, SF-00290 Helsinki, Finland

J Ignatius

Correspondence to: Professor Davies.

Received 29 August 1995 Revised version accepted for publication 25 October 1995 depends on the degree of respiratory muscle weakness. The age of onset in type III SMA is very variable, ranging from infancy to early adulthood. Disease progression is slow and these patients may remain ambulant for decades. No clues to the molecular basis of cell death in SMA have yet emerged and it is anticipated that characterisation of the disease at the molecular genetic level will open the way to its elucidation.

We, and others, have shown that all three forms of the disease map to chromosome $5 q 11.2-13.3 .^{5-8}$ The disease mutation has been further refined by the identification of microsatellites that show strong linkage disequilibrium with the disease..$^{-13}$ These studies indicate a high frequency of null alleles associated with the SMA phenotype suggesting that deletions of the critical region are common in patients. Two candidate genes have been reported. The survival motor neurone gene (SMN) exists in two almost identical copies in $5 \mathrm{q} 13$, and the telemoric copy is deleted in $98.6 \%$ of patients. ${ }^{14}$ Three patients without deletions have point mutations. We have confirmed the SMN deletion frequency and also shown that these deletions arise de novo. ${ }^{15}$ The neuronal apoptosis inhibitory protein (NAIP) gene, which lies in the region adjacent to the SMN gene, is deleted in $45 \%$ of patients with type I SMA and $18 \%$ of those with types II and III. ${ }^{16}$ These authors reported homozygous deletions in $3 / 168$ carriers. Pseudogenes of NAIP are present in multiple copies in the region and the deletions may be complex. A third transcript, XS2G3, mapping to the candidate region has been shown to be deleted in patients with SMA, ${ }^{17}$ but subsequently has been shown to correspond to exon 7 of NAIP in the opposite orientation. XS2G3 therefore detects the same deletions as NAIP. ${ }^{18}$

The physical maps of the SMA region presented as cloned contigs ${ }^{1019-22}$ are not consistent, making the determination of the physical organisation of the candidate genes difficult. ${ }^{23}$ The presence of multicopy repetitive sequences, pseudogenes, and retrotransposonlike sequences underlies the unstable nature of this area of the genome. ${ }^{2024-26}$

In view of the complexity of the region and the lack of correlation between the SMN gene deletions and the phenotype, we have attempted to construct a deletion genotype for the disease. Here we present analysis of deletions in NAIP and SMN genes in a large number of SMA patients and carriers. Our data suggest that more extensive deletions are associated with more severe disease. 


\section{Materials and methods}

All patients conformed to the consensus diagnostic criteria of the International SMA Consortium. ${ }^{4}$ Subjects were drawn from ethnically diverse populations in the $\mathrm{UK}$ and included at least $70 \%$ of the known living cases of SMA ever diagnosed in Finland. Informed consent was obtained from all families participating in this study. Control DNA samples consisted of patients with haemoglobinopathies, polycystic kidney disease, and fragile $\mathrm{X}$ syndrome from the UK and elsewhere in Europe, and also included samples drawn from healthy blood donors from the Finnish population.

A total of 187 families with SMA were used for this analysis. Where there were multiple affected members in a family, only one subject contributed to the data set although all were analysed. In families with differences in clinical severity of the disease, the phenotype of the first born affected was used since the genotype for both phenotypes was identical. Among these families, five were of Finnish origin and two were of UK origin. In all cases it was the association of type II with type III that was observed. The association of type I SMA with the milder forms was not observed. Carrier status was assumed for parents and was determined by linkage analysis for sibs. There were 77 families with type I SMA, 69 families with type II SMA, and 41 families with type III SMA.

SSCP ANALYSIS OF SMN EXONS 7 AND 8

SSCP analysis was carried out as described by Lefebvre et $a l^{14}$ but with the modification of using ${ }^{35} \mathrm{~S}$ labelled dATP. ${ }^{15}$ Genomic DNA (100-200 ng) was amplified using exon 7 (R111 and 541C770) and exon 8 (541C1120 and 541C960) specific oligonucleotide primers.

PCR ANALYSIS OF NAIP EXONS 5 AND 13 Genomic DNA (100-200 ng) was amplified with NAIP exon 5 (1863 and 1864) and exon 13 (1258 and 1343) specific oligonucleotide primers in a multiplex PCR reaction as described by Roy et al. ${ }^{16}$ Exon 13 is present in both functional and pseudogene copies of NAIP and therefore can be used as a positive PCR control for exon 5 which is present only in the functional NAIP gene. PCR was carried out using standard cycles with an annealing temperature of $60^{\circ} \mathrm{C}$. Samples were loaded on to a $1 \cdot 2 \%$ agarose gel containing ethidium bromide. Electrophoresis was carried out at $80-100 \mathrm{~V}$ for 1.5 hours to resolve both exon 5 and exon 13 products, which were visualised under UV fluorescence. All results showing deletions in
NAIP exon 5 were repeated with exon 5 primers only, as we have found that occasionally the multiplex reaction led to inhibition of amplification of one or other exon.

\section{Results}

ANALYSIS OF DELETIONS IN THE SMN GENE The analysis of SMN in 140/187 of these families has been previously reported. ${ }^{15}$ Absence of exons 7 and 8 specific to telomeric SMN was used to identify deletions of the SMN gene. A total of $175 / 187$ patients $(93.5 \%)$ were homozygously deleted for at least part of the telomeric copy of SMN and there was no significant difference in deletions seen in the different forms of the disease (74/77 type I, 64/ 69 type II, 37/41 type III). Of these patients, $88.1 \%$ had deletions of both exons 7 and 8 , and $5 \cdot 4 \%$ had only exon 7 deletions. None was deleted for exon 8 only. Of the patients not deleted for SMN, all but two exhibited no homozygous deletions, possessing at least one copy of NAIP and both the centromeric and telomeric forms of SMN. One patient with type I SMA showed no deletions in SMN but was deleted for NAIP exon 5 (see below). The other patient, with type II SMA, was homozygously deleted for the centromeric copies of exons 7 and 8 but retained the telomeric copies. This pattern was observed in 19/358 normal subjects, but in none of the carriers. The combination of deletions in both the centromeric and telomeric copies of SMN was never observed, suggesting that this might represent a lethal mutation. Of the 373 carriers screened, none was homozygously deleted for SMN. Of the 31 normal sibs identified by linkage studies as being non-carriers, none had any deletions. Of the 358 normal controls analysed, none showed homozygous deletions of the telomeric SMN gene.

ANALYSIS OF DELETIONS IN THE NAIP GENE

Exon 5 of NAIP, which is specific for the functional NAIP gene, was deleted in 51/77 of patients with type I SMA $(66 \cdot 2 \%)$ but only deleted in $7 / 69$ of type II and 5/41 of type III patients $(10 \cdot 2 \%$ and $12 \cdot 2 \%$, respectively). This is a higher proportion of deletions than that found by Roy et $a l^{16}$ (45\% of type I patients). This increase cannot, in our data set, be accounted for by the Finnish population in which the deletion rate was $44 \%$. Of the 373 carriers tested, seven were deleted for exon 5 of NAIP. Of these deleted carriers, 6/131 were parents of type I SMA patients, 0/111 was a parent of a type II patient, and 1/131 was a parent of a

Deletion genotypes including SMN and NAIP

\begin{tabular}{|c|c|c|c|c|c|c|}
\hline Phenotype & $\begin{array}{l}\text { Genotype A } \\
\text { del del del }\end{array}$ & $\begin{array}{l}\text { Genotype B } \\
\text { del del } n\end{array}$ & $\begin{array}{l}\text { Genotype C } \\
\operatorname{del} n n\end{array}$ & $\begin{array}{l}\text { Genotype } D \\
\text { del } n \text { del }\end{array}$ & $\begin{array}{l}\text { Genotype } E \\
n n \text { del }\end{array}$ & $\begin{array}{l}\text { Genotype } F \\
n n n\end{array}$ \\
\hline $\begin{array}{l}\text { Type I } \\
\text { Type II } \\
\text { Type III } \\
\text { Carriers }\end{array}$ & $\begin{array}{cl}49 / 77 & (63 \cdot 6 \%) \\
6 / 69 & (8 \cdot 7 \%) \\
5 / 41 & (12 \cdot 2 \%) \\
0 / 373 & (0 \%)\end{array}$ & $\begin{array}{cl}23 / 77 & (29 \cdot 9 \%) \\
51 / 69 & (73 \cdot 9 \%) \\
30 / 41 & (73 \cdot 2 \%) \\
0 / 373 & (0 \%)\end{array}$ & $\begin{array}{ll}1 / 77 & (1 \cdot 3 \%) \\
6 / 99 & (8 \cdot 7 \%) \\
2 / 41 & (4 \cdot 8 \%) \\
0 / 373 & (0 \%)\end{array}$ & $\begin{array}{ll}1 / 77 & (1 \cdot 3 \%) \\
1 / 69 & (1 \cdot 4 \%) \\
0 / 41 & (0 \%) \\
0 / 373 & (0 \%)\end{array}$ & $\begin{array}{ll}1 / 77 & (1 \cdot 3 \%) \\
0 / 69 & (0 \%) \\
0 / 41 & (0 \%) \\
7 / 373 & (1.9 \%)\end{array}$ & $\begin{array}{cc}2 / 77 & (2 \cdot 6 \%) \\
5 / 69 & (7 \cdot 3 \%) \\
4 / 41 & (9 \cdot 8 \%) \\
366 / 373 & (98 \cdot 1 \%)\end{array}$ \\
\hline
\end{tabular}

del $=$ deleted.

$\mathrm{n}=$ not deleted. 

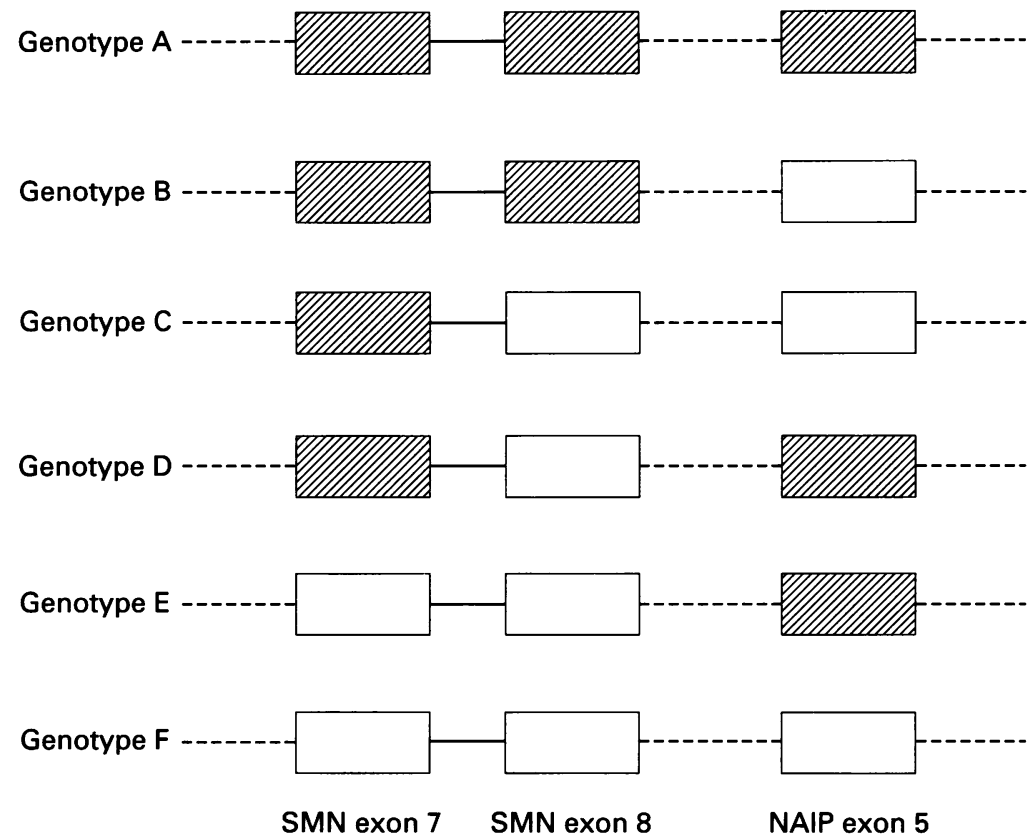

A diagrammatic representation of the deletion genotypes described in the text. Hatched boxes represent deleted exons. Owing to the polymorphic nature of the region no particular physical orientation of SMN and NAIP is assumed.

type III patient. The 31 normal sibs showed no deletions. None of the 532 normal controls tested was deleted for NAIP.

COMBINING SMN AND NAIP GENES TO PRODUCE A DELETION GENOTYPE

The frequency of deletions of both SMN and NAIP genes in patients, carriers, and normal controls is shown in the table. These genotypes are depicted schematically in the figure. For simplicity, the order of the genes is assumed to be centromere-SMN (cen)-SMN (tel)NAIP-telomere, as published by Lefebvre et $a l .{ }^{14}$ This does not rule out the possibility that in some subjects, such as those with genotype $\mathrm{D}$, the order could be reversed relative to the telomere, as published by Roy et al. ${ }^{16}$ It is not assumed that the deletions are contiguous. The deletions represented by genotype $A$ would include telomeric SMN exon 7 and exon 8, and also NAIP (del del del). Genotype B represents a deletion including and proximal to telomeric SMN exon 7 and exon 8 only (del del n). Genotype $\mathrm{C}$ represents a deletion including and proximal to telomeric SMN exon 7 only (del $\mathrm{n} \mathrm{n}$ ). Genotype $\mathrm{D}$ represents a deletion in telomeric SMN exon 7 and NAIP only (del $n$ del).

Genotype E represents a deletion for NAIP only ( $\mathrm{n}$ del). Genotype $\mathrm{F}$ has no detected deletions for telomeric SMN exon 7, SMN exon 8 , or NAIP exon 5 ( $n n n$ ). Since heterozygously deleted subjects cannot be determined using these assays it cannot be excluded that more extensive deletions (genotype A) exist on one of the two chromosomes of an affected subject who, for example, has genotype $\mathrm{B}$ or $\mathrm{C}$.

Genotype A, which represents a larger deletion, has a higher incidence in type I patients compared with types II and III $\left(\chi^{2}{ }_{4}=59 \cdot 95\right.$, $\mathrm{p}<0 \cdot 000001)$. There is no statistically significant difference between the two milder forms. Genotypes B and C which represent smaller deletions are predominantly found in type II and III patients. Genotype D was found in two patients and could be explained either by two deletion events or rearrangement of NAIP placing it proximal to SMN according to the map of Roy et al. ${ }^{16}$ Genotype E, which represents the smallest deletion, was found in one type I patient, but also in 11 carriers. Genotype F, with no detectable deletions, is more frequent in types II and III than type I.

In analyses of multiply affected sibships including those with a difference in the clinical severity of the disease, the affected sibs had identical deletions when assayed using primers for exon 5 of NAIP and exons 7 and 8 of SMN.

\section{Discussion}

This is an extensive analysis of the incidence of deletions in both SMN and NAIP genes in a large set of SMA patients and carriers. In the original papers, SMN gene deletions were found not to correlate with the severity of the phenotype whereas deletions in NAIP occurred more frequently in the more severely affected type I patients. ${ }^{1416}$ The data presented here suggest that there is a very strong correlation between the extent of the deletions and the severity of the disease.

The incidence of deletions which include both SMN and NAIP genes in SMA patients was much higher in type I patients compared to type II and III patients $(63.6 \%, 8.8 \%$, $12 \cdot 2 \%$, respectively). Patients suffering from the milder forms of the disease showed smaller deletions which predominantly included SMN but not NAIP. There is no statistically significant difference between deletions in types II and III suggesting that these two subtypes of SMA may represent a continuum. Consistent with this observation is the fact that families with two different phenotypes rarely include subjects with type I SMA. Furthermore, while the Finnish population might seem to contain a larger than expected number of mixed phenotype families, these are the families for which we have the most accurate clinical data and it is therefore likely that the true incidence of mixed families is higher than reported here.

In two patients (type I and type II) who had deletions of SMN exon 7 and NAIP exon 5, but no deletion of $S M N$ exon 8 , it is possible that NAIP is proximal to SMN in accordance with the map published by Roy et al, ${ }^{16}$ or that there are two deletion events. One type I patient is not deleted for SMN but deleted for NAIP, a genotype seen in $1.9 \%$ of carriers. This patient may possess other mutations in either SMN or other genes in the region. We are currently exploring this in detail. It is of note that deletions of NAIP in carriers of SMA occur almost exclusively in parents of type I patients. The detection of NAIP deletions in carriers suggests that the non-mutated allele is probably deleted frequently. This also confirms the high degree of instability of this region. The patients who 
did not show any detectable deletions of the two genes are being characterised further for the presence of point mutations.

The very high frequency of deletions in the SMN gene suggests that mutations in SMN are directly involved in the disease or that the gene lies very close to the critical region. NAIP may also be involved in the pathogenesis of SMA either alone or by directly interacting with the SMN gene product. ${ }^{1623}$ The data presented in this paper strongly suggest that homozygous deletion of a portion of the SMN gene is clearly associated with the SMA phenotype, both in the presence and absence of homozygous deletions of NAIP. However, since both SMN and NAIP genes are present in multiple copies in this region, an alternative explanation could be that the copy number of these genes is important in the manifestation of the disease. This idea is supported by the genotype analysis using closely linked microsatellites which are also present in more than one copy in the region. There is a strong correlation between the severity of the phenotype and the number of alleles observed. ${ }^{101327}$ The extent of deletions on each of the chromosomes could influence the amount of gene product. Thus the combination of genotype $A$ and $B$ on the two chromosomes of a patient could result in a more severe phenotype than genotype B and B combined. It is also important to emphasise that the current methods of analysis of both SMN and NAIP do not allow quantitation of the number of copies of these genes. While we refer to deletions occurring in SMN, it is theoretically possible that gene conversion events could account for the observed genotypes, for example, by the replacement of the telomeric version of SMN with portions of the centromeric sequence.

The assays of exon deletions used in our analyses are not sensitive enough to detect the precise extent of the deletions or to differentiate between in frame or out of frame deletions. Thus it is possible that the deletions encompass larger isoforms of NAIP or SMN, or disrupt other genes in the region. For example, the DMD locus encodes a ubiquitously expressed small $4.8 \mathrm{~kb}$ transcript, but the larger $14 \mathrm{~kb}$ transcript expressed predominantly in muscle determines the phenotype. ${ }^{28}$

In summary, the data presented here confirm that SMA is associated with a high frequency of deletions in chromosome 5q13 and indicate that the more severe phenotype is associated with more extensive deletions. If the molecular basis of SMA is to be understood it is vital that a coherent picture of the physical organisation of these genes emerges and the relationship between SMN and NAIP defined. To that end the above data will provide initial clues to the extent of deletions at the physical level and may aid the ordering of genetic loci.

We thank M A Nesbit and P McShane for helpful discussions. We are also grateful to the SMA International Consortium members who have shared ideas and resources with us. We are grateful to the Medical Research Council, the Muscular Dystrophy Group of Great Britain and Northern Ireland, and yystrophy Group of Great Britain and Northern Ireland, and the Muscular Dystrophy Group, USA for financial support. We $\mathrm{S}$ Knight, and J Old for normal control samples.

1 Dubowitz V. Muscle disorders of childhood. London: Saunders, 1978:146-90.

2 Braun S, Croizat B, Lagrange MC, Warter JM, Poindron $P$. Constitutive muscular abnormalities in culture in spinal muscular atrophy. Lancet 1995;345:694-5.

3 Pearn JH. Classification of spinal muscular atrophies. Lancet 1980;i:919-22.

4 Munsat TL, Davies KE. Workshop Report. International SMA collaboration. Neuromusc Disord 1992;2:423-8.

5 Brustowicz LM, Lehner T, Castilla LH, et al. Genetic mapping of chronic childhood onset spinal muscular atrophy to chromosome 5p11.2-13.3. Nature 1990;344:5401 .

6 Gilliam TC, Brustowicz LM, Castilla LH, et al. Genetic homogeneity between acute and chronic forms of spinal muscular atrophy. Nature 1990;345:823-5.

7 Melki J, Abdelhak S, Sheth P, et al. Gene for chronic proximal spinal muscular atrophies maps to chromosome 5q. Nature 1990;344:767-8.

8 Melki J, Sheth P, Abdelhak S, et al. Mapping of acute (type I) spinal muscular atrophy to chromosome 5q12-q14. Lancet 1990;336:271-3.

9 Burghes AHM, Ingraham SE, McLean M, et al. A multicopy dinucleotide repeat marker that maps close to the spina muscular atrophy gene. Genomics 1994;21:394-402.

10 Melki J, Lefebvre S, Burglen L, et al. De novo and inherited deletions of the $5 \mathrm{q} 13$ region in spinal muscular atrophies. Science 1994;264:1474-7.

11 McLean MD, Roy N, MacKenzie AW, et al. Two 5q13 simple tandem repeat loci are in linkage disequilibrium simple tandem repeat loci are in linkage disequilibrium with type I

12 Daniels RJ, Campbell L, Rodrigues NR, et al. Genomic rearrangements in childhood spinal muscular atrophy: linkage disequilibrium with a null allele. $\mathcal{F}$ Med Genet 1995;32:93-6.

13 DiDonato CJ, Morgan K, Carpten JD, et al. Association between $\mathrm{AgClCA}$ alleles and severity of autosomal recessive proximal spinal muscular atrophy. Am $\mathcal{F}$ Hum Genet 1994;55:1218-29.

14 Lefebvre S, Burglen L, Reboullet S, et al. Identification and characterisation of a spinal muscular atrophy determining gene. Cell 1995;80:155-65.

15 Rodrigues NR, Owen N, Talbot K, et al. Deletions in the survival motor neuron gene on $5 \mathrm{q} 13$ in autosomal recessive survival motor neuron gene on 5q13 in autosomal recessive

16 Roy N, Mahadevan MS, McLean M, et al. The gene for neuronal apoptosis inhibitory protein is partially deleted in individuals with spinal muscular atrophy. Cell 1995;80: 167-78.

17 Thompson TG, DiDonato CJ, Simard LR, et al. A novel cDNA detects homozygous microdeletions in greater than $50 \%$ of type I spinal muscular atrophy patients. Nature Genet 1995;9:56-62.

18 Mahadevan MS, Korneluk RG, Roy N, MacKenzie A, Ikeda JE. SMA genes: deleted and duplicated. Nature Genet 1995;9:112-13.

19 Kleyn PW, Wang CH, Lien LL, et al. Construction of a yeast artificial chromosome contig spanning the spinal muscular atrophy disease gene region. Proc Natl Acad Sci muscular 1993;90:6801-5.

20 Francis MJ, Morrison KE, Campbell L, et al. A contig of non-chimaeric YACs containing the spinal muscular of non-chimaeric YACs containing the spinal muscular

21 Carpten JD, Didonato CJ, Ingraham SE, et al. A YAC contig Carpten JD, Didonato CJ, Ingraham SE, et al. A YAC contig
of the region containing the spinal muscular atrophy gene (SMA): identification of an unstable region. Genomics 1994;24:351-6.

22 van der Steege G, Draaijers TG, Grootscholten PM, et al. A provisional transcript map of the spinal muscular atrophy (SMA) critical region. Eur $\mathcal{F}$ Hum Genet 1995;3:87-95.

23 Lewin B. Genes for SMA: multum in parvo. Cell 1995;80: $1-5$

24 Sargent CA, Chalmers IJ, Leversha M, Affara NA. A rearrangement on chromosome 5 of an expressed human $\beta$-glucuronidase pseudogene. Mamm Gen 1994;5:791-6.

25 Theodosiou AM, Morrison KE, Nesbit AM, et al. Complex repetitive arrangements of gene sequence in the candidate f Hum Genet 1994;55:1209-17.

26 Francis MJ, Nesbit MA, Theodosiou AM, et al. Mapping of retrotransposon sequences in the unstable region surof retrotransposon sequences in the unstable region sur-
rounding the spinal muscular atrophy locus in 5q13. rounding the spinal muscu.

27 Wirth B, Hahnen E, Morgan K, et al. Allelic association and deletions in autosomal recessive proximal spinal muscular atrophy: association of marker genotype with disease severity and candidate cDNAs. Hum Mol Genet 1995;8: 1273-84.

28 Tinsley JM, Blake DJ, Pearce $M$. Dystrophin and related proteins. Curr Opin Genet Dev 1993;3:484-90. 